\title{
Experimental generation and observation of a super-resolution optical tube
}

\author{
Jianghua $\mathrm{Xu}^{*}$, Liping Jiang ${ }^{\dagger}$, Houfei $\mathrm{Zhu}^{*}$, Lingling $\mathrm{Liu}^{\S}$, Jinbing $\mathrm{Hu}^{\Uparrow}$, \\ Haifeng Wangll and Songlin Zhuang** \\ Institute of Imaging Optics, Ministry of Education and Shanghai Key Lab \\ of Modern Optical Systems \\ University of Shanghai for Science and Technology \\ Shanghai 200093, P. R. China \\ *a_peasant@easy.com \\ †24715713@qq.com \\ \$911670318@qq.com \\ \$liuling0543@126.com \\ Thujinbing@usst.edu.cn \\ "wanghaifeng@usst.edu.cn \\ **slzhuang@yahoo.com
}

Received 13 December 2015

Accepted 20 January 2016

Published 30 March 2016

\begin{abstract}
We generated a super-resolution optical tube by tightly focusing a binary phase modulated azimuthally polarized laser beam. The binary phase modulation is achieved by a glass substrate with multi-belt concentric ring grooves. We also characterized the 3D beam profile by using a crossshaped knife-edge fabricated on a silicon photo-detector. The size of the super-resolution dark spot in the tube is $0.32 \lambda$, which remains unchanged for $\sim 4 \lambda$ within the tube. This optical tube may find applications in super-resolution microscopy, optical trapping and particle acceleration.
\end{abstract}

Keywords: Laser beam shaping; photodetectors; binary optics; fluorescence microscopy; polarization.

Super-resolution focused light spots are always expected in scanning optical microscopy. There are bright focused light spots and dark focused spots in stimulated emission depletion (STED) microscopy,$^{1-3}$ and the two kinds of spots have different light frequencies. For obtaining nanosized effective light spot, the bright spot acts as the excitation beam to excite the fluorescent molecules and the dark spot acts as the STED beam to de-excite the marginal fluorescent molecules. However, the size of effective

${ }$ Corresponding author.

This is an Open Access article published by World Scientific Publishing Company. It is distributed under the terms of the Creative Commons Attribution 4.0 (CC-BY) License. Further distribution of this work is permitted, provided the original work is properly cited. 
light spot depends largely on the parameters of the dark focused spot. To reduce the size of focused bright light spots and obtain super-resolution, some researchers used binary optics to modulate the phase of light field on the aperture of the condensing lens. $^{4-6}$

In this experiment, we apply a binary optics to the aperture of a focusing lens to obtain an optical tube with super-resolution dark spot, which may lead to a much higher resolution in STED microscopy. This super-resolution dark spot is achieved by tightly focusing an azimuthally polarized BesselGaussian laser beam with a combination of a binary optics and a high-NA lens. We get the optimized parameters of the binary optics, and the results of modulating the phase by the binary optics are not only reducing the size of dark focused spot but also enhancing the depth of the focus. This subwavelength focused dark spot makes the STED microscopy achieve higher super-resolution while the three-dimensional scanning would be much more easy to access with the longer focal depth. It will be significant to the researches of the biomedicine and the molecular structure. Also it makes the optical tube become the more ideal STED beam. ${ }^{7,8}$

Following the theory of Richards and Wolf, ${ }^{9}$ and the earlier works, ${ }^{10-13}$ the electric field near the focal plane of an azimuthally polarized Bessel-Gaussian beam focused by a high-NA lens can be expressed in the following form:

$$
\begin{aligned}
& E_{\varphi}(r, z) \\
& \quad=2 A \int_{0}^{\alpha} \cos ^{1 / 2} \theta \sin \theta l(\theta) J_{1}(k r \sin \theta) e^{i k z \cos \theta} d \theta,
\end{aligned}
$$

where $\theta$ is the tangential angle with respect to the $z$-axis, $\alpha=\arcsin (N A)$, and the origin is $r=0, z=0$ located at the focal point position. $k=2 \pi / \lambda$ is the wave number, $J_{1}(x)$ is the first-order Bessel function of the first kind and $l(\theta)$ is the amplitude distribution of the azimuthally polarized BesselGaussian beam, which is given as

$$
l(\theta)=\exp \left[-\beta^{2}\left(\frac{\sin \theta}{\sin \alpha}\right)^{2}\right] J_{1}\left(2 \gamma \frac{\sin \theta}{\sin \alpha}\right),
$$

where $\beta$ and $\gamma$ are parameters which we take as unity in our configuration. It is similar to illuminating with a Laguerre-Gaussian beam. Here, we use a focusing lens with the numerical aperture of 0.95 $\left(\alpha \approx 71.8^{\circ}\right)$. In this case, the dark focused spot has a full width of the half maximum (FWHM) as large as $0.4 \lambda$ and the length of nondiverging region is $4 \lambda$.

To obtain an optical tube that has smaller dark spot and longer focal depth, we add a tailor-made binary optical element on the focal lens aperture. This optical element is a glass substrate with fivebelt concentric ring grooves of which the changes in phase are $0-\pi$ alternations, and the radius $r_{i}=$ $\sin \theta_{i} / N A$ corresponds to the angle of $\theta_{i}$. Then the function $l(\theta)$ in Eqs. (1) and (2) should be replaced by the function $T(\theta) l(\theta)$, where $T(\theta)$ is the transmission-function ${ }^{14}$ :

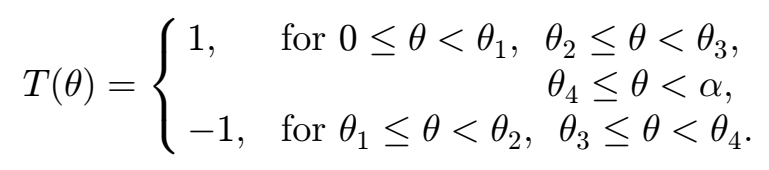

We optimized the parameters to obtain the optical tube of perfection, and we suggest the set of angles found from reasonable optimizations of all parameters as an example, the corresponding positions of $\mathrm{r}_{i}$ and $\theta_{i}$ are shown in Table 1 .

The electric field intensity distribution in focus for this case is shown in Fig. 1(b). The FWHM of the dark focused spot in Fig. 1 (b) is $0.32 \lambda$, which is smaller than that obtained without the binary optics shown in Fig. 1(a) $(\mathrm{FWHM}=0.4 \lambda)$.

For the detection, two methods of measuring the focal spot size have been reported so far: knife-edge scanning ${ }^{15,16}$ and a photo-resist exposure method. ${ }^{17}$ We employed the knife-edge scanning method in this study because it gives spot size as a function of focal depth. For this knife-edge method, we used a photo-diode to measure the photocurrent change as a function of the knife-edge position by cutting the focused beam spot with the sharp cross-shape of a metal blade. The structure of the cross-shaped knife-edge detector is shown in Fig. 2(b). The photo-diode is fabricated by combining the $p$-GaAs and the substrate of $n$-GaAs and produces the depletion layer between $p$-GaAs and $n$-GaAs. And then the $\mathrm{Au} / \mathrm{Ti}$ is coated on the surface of $p$-GaAs. There is a cross-shaped knife-edge in the $\mathrm{Au} / \mathrm{Ti}$ film. This detector is set on a nanometer platform moving in three-dimension direction.

Table 1. Optimized parameters of binary optics.

\begin{tabular}{llccc}
\hline $\mathrm{i}$ & 1 & 2 & 3 & 4 \\
\hline$\Theta($ degree $)$ & 4.96 & 21.79 & 34.25 & 46.87 \\
$r\left(r_{0}\right)$ & 0.091 & 0.391 & 0.592 & 0.768 \\
\hline
\end{tabular}




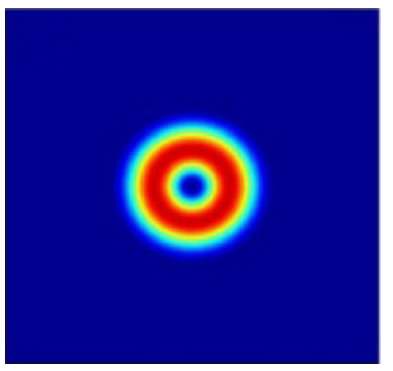

(a)

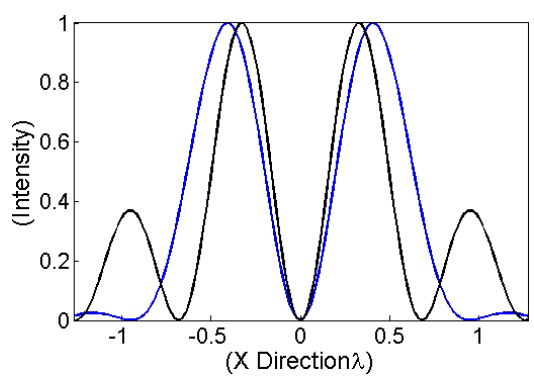

(c)

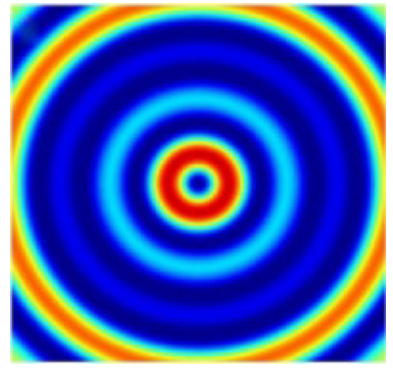

(b)
Fig. 1. (Color online) (a) Intensity distribution in focus without the binary optics. (b) Intensity distribution in focus with the binary optics. (c) Cross-section of intensity distribution with (black line) and without (blue line) the binary optics.

The experiment setup is shown in Fig. 2(a). The transversal linear polarized beam is projected by a $\mathrm{He}-\mathrm{Ne}$ laser and passed through a collimating lens, then reflected by a mirror set at an angle of $45^{\circ}$ respect to the optical axis, and the laser beam became an azimuthally polarized beam after modulated by the polarization converter $(\mathrm{PC}),{ }^{18}$ then it

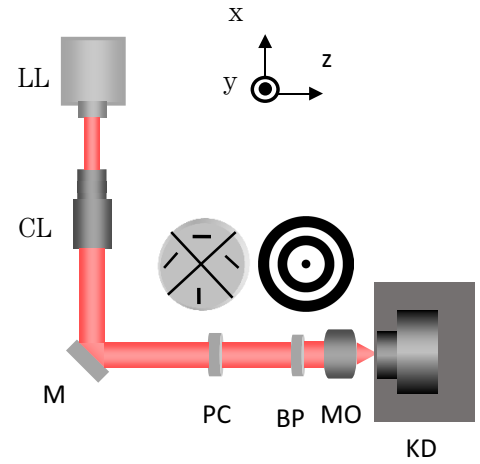

(a)

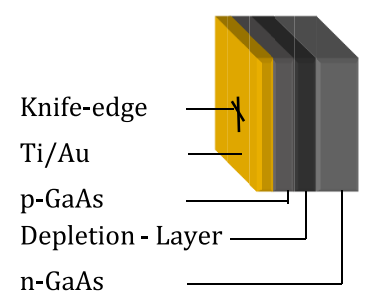

(b)
Fig. 2. Schematic experiment setup. (a) LL, linear polarized laser; CL, collimating lens; M, mirror; PC, polarization converter; $\mathrm{BP}$, binary optics; $\mathrm{MO}$, microscope objective $(\mathrm{NA}=$ 0.95); KD, knife-edge detector set on a nanometer-platform (gray region). (b) The cross-shaped knife-edge detector. will be tightly focused by a hybrid lens which has an additional binary optics on its aperture, and detected by the knife-edge detector that sets on a nanometer platform. The PC is fabricated by gluing four segmented half-wave plates together, and the optical axis of these four half-wave plates are set at particular angles as shown in Fig. 2(a). The binary optics we designed is achieved by electron-beam lithography on a glass substrate. Thus, we can get the electric field intensity of all the points near the focus plane by shifting the cross-shaped knife-edge detector in three-dimension direction. We reconstructed the intensity distribution of the dark spot in $x-z$ plane that is shown in Fig. 3(b), and it got a perfect match with the calculated result in theory as shown in Fig. 3(a).

As it is shown in Fig. 3(c), the focused azimuthally polarized beam is circular symmetry, and hence the electric intensity distribution in the $y-z$ plane is the same as the $x-z$ plane. The length of this optical tube which is the distance of the nondiffraction region is $4 \lambda$, and the radius of the central tube (FWHM) is $0.32 \lambda$, it is smaller than the diffraction limit for this focusing lens $\lambda /(2 N A)=$ $0.526 \lambda$. In addition, the centric ring of the hollow optical tube has seized about $56.9 \%$ of energy of the electric field in the focus while the sidelobes have

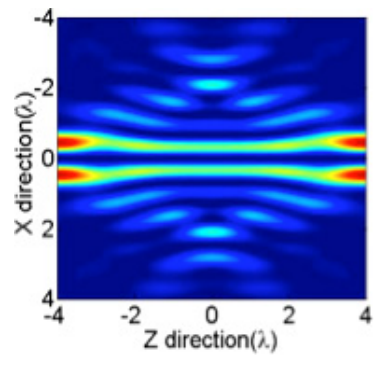

(a)

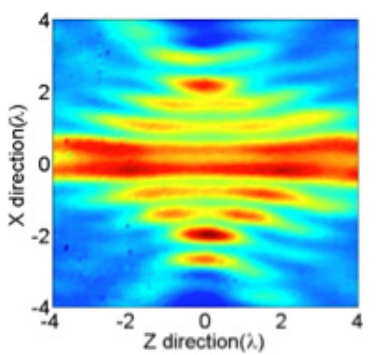

(b)

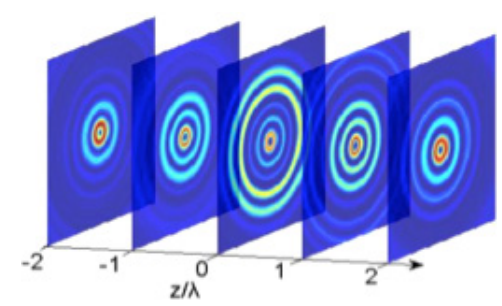

(c)

Fig. 3. The electric intensity distribution in the $x-z$ plane after the phase modulation of the binary optics. (a) Calculated result. (b) Experimental result. (c) Cross-sectional of the beam at different positions along $z$-axis. 
high divergence, and there are also some methods to eliminate the sidelobe effects, ${ }^{19,20}$ thus we can find application to use this super-resolution optical tube feasibly in comparatively higher utility efficiency.

In conclusion, we have generated and observed a super-resolution optical tube in experiment by tightly focusing a binary phase modulated azimuthally polarized laser beam. The binary phase modulator we used here can not only reduce the size of the focused dark spot to achieve super-resolution but also enhance the depth of the focus. Compared with the focused azimuthally polarized laser beam without using the binary optics, it could increase the resolution of the STED microscopy by $25 \%$ with the same intensity, or reach the same resolution with a lower intensity which is quite significant to the application of the STED microscopy in biomedical research for that the laser with higher intensity may injure cells.

\section{Acknowledgments}

National 973 Program of China (2015CB352001); The Program for Professor of Special Appointment (Eastern Scholar) at Shanghai Institutions of Higher learning.

\section{References}

1. X. Hao, C. Kuang, T. Wang et al., "Effects of polarization on the de-excitation dark focal spot in STED microscopy," J. Opt. 12(11): 115707 (115707).

2. H. Wang, J. R. Sheppard, K. Ravi, S. T. Ho, G. Vienne, "Fighting against diffraction: Apodization and near field diffraction structures," Laser Photonics Reviews 6(3), 354-392 (2012).

3. Y. Xue, C. Kuang, S. Li et al., "Sharper fluorescent super-resolution spot generated by azimuthally polarized beam in STED microscopy," Opt. Express 20(16), 17653-17666 (2012).

4. H. Wang, F. Gan, "High focal depth with a purephase apodizer," Appl. Opt. 40(31), 5658-5662 (2001).

5. D. M. de Juana, V. F. Canales, P. J. Valle, M. P. Cagigal, "Focusing properties of annular binary phase filters," Opt. Commun. 229(1), 71-77 (2004).

6. H. Guo, X. Weng, M. Jiang, et al., "Tight focusing of a higher-order radially polarized beam transmitting through multi-zone binary phase pupil filters," Opt. Express 21(5), 5363-5372 (2013).

7. S. W. Hell, J. Wichmann, "Breaking the diffraction resolution limit by stimulated emission: Stimulated emission-depletion fluorescence microscopy," Opt. Letters 19(11), 780-782 (1994).

8. D. Wildanger, E. Rittweger, L. Kastrup, S. W. Hell, "STED microscopy with a supercontinuum laser source," Opt. Express 16(13), 9614-9621 (2008).

9. B. Richards, E. Wolf, "Electromagnetic Diffraction in Optical Systems. II. Structure of the Image Field in an Aplanatic System," P. Roy. Soc. Lond. A Mat. 253(1274), 358-379 (1959).

10. Z. Bouchal, M. Olivík, "Non-diffractive vector Bessel beams," J. Modern Opt. 42(8), 1555-1566 (1995).

11. K. Youngworth, T. Brown, "Focusing of high numerical aperture cylindrical-vector beams," Opt. Express 7(2), 77-87 (2000).

12. Q. Zhan, "Focus shaping using cylindrical vector beams," Opt. Express 10(7), 324 (2002).

13. S. Sato, Y. Kozawa, "Hollow vortex beams," JOSA A, 26(1), 142-146 (2009).

14. H. Wang, L. Shi, B. Lukyanchuk et al., "Creation of a needle of longitudinally polarized light in vacuum using binary optics," Nature Photon. 2(8), 501-505 (2008).

15. R. Dorn, S. Quabis, G. Leuchs, "The focus of lightlinear polarization breaks the rotational symmetry of the focal spot," J. Modern Opt. 50(12), 19171926 (2003).

16. K. Kitamura, K. Sakai, S. Noda, "Sub-wavelength focal spot with long depth of focus generated by radially polarized, narrow-width annular beam," Opt. Express 18(5), 4518-4525 (2010).

17. B. Hao, J. Leger, "Experimental measurement of longitudinal component in the vicinity of focused radially polarized beam," Opt. Express 15(6), 35503556 (2007).

18. Q. Zhan, "Cylindrical vector beams: From mathematical concepts to applications," Adv. Opt. Photon. 1(1), 1-57 (2009).

19. Y. Zha, J. Wei, F. Gan, "Performance improvement of super-resolving pupil filters via combination with nonlinear saturable absorption films," Opt. Commun. 293, 139-148 (2013).

20. Y. Zha, J. Wei, F. Gan, "A novel design for maskless direct laser writing nanolithography: Combination of diffractive optical element and nonlinear absorption inorganic resists," Opt. Commun. 304, 49-53 (2013). 